\title{
Tunneling Rates of Single Electrons on Liquid Helium in an Extracting Field
}

\section{Ismail Karakurt}

Published online: 28 May 2009

(C) Springer Science+Business Media, LLC 2009

\section{Erratum to: Low Temp Phys (2009) 154: 77-84 \\ DOI 10.1007/s10909-008-9852-0}

There is an error in the prefactor of the fitting function in Eq. (6) of the original article. The correct form includes the square root of $p(z)$ instead of $p(z)$. The asymptotic amplitude obtained from a given fit is larger with correct form of the prefactor. However, the same tunneling rate is obtained, and both the phase and the form of the lineshape (i.e., the linewidth) do not change since the fitting is done far away from the surface and over just a wavelength.

The online version of the original article can be found under doi:10.1007/s10909-008-9852-0.

I. Karakurt (凶)

Department of Physics, Isik University, Kumbaba Mevkii, Sile, 34980, Istanbul, Turkey e-mail: ikarakurt@isikun.edu.tr 